\section{Finanzierungsfragen des Pervasive Computing im Gesundheitswesen}

\author{
von Carsten Orwat und Veneta Panova, \\ ITAS
}

Im Gesundheitswesen haben die Möglichkeiten der Kostenerstattung und der Refinanzierung von medizinischen Leistungen starke Wirkungen darauf, wie sich technische Innovationen durchsetzen. In diesem Artikel werden die Finanzierungsmöglichkeiten und damit die Innovations- und Diffusionsbedingungen für Pervasive Computing bzw. Ubiquitous Computing betrachtet. Aufgrund einiger Besonderheiten des Pervasive Computing kann davon ausgegangen werden, dass die Umsetzungsbarrieren, die bereits im Allgemeinen für Informationsund Kommunikationstechnologien im Gesundheitswesen bestehen, für Pervasive Computing noch höher einzuschätzen sind.

\section{Pervasive-Computing-Systeme im Gesundheitswesen}

Pervasive Computing, das hier synonym zu verwenden ist mit Ubiquitous Computing oder Ambient Intelligence, tritt in höchst unterschiedlichen Anwendungen im Gesundheitswesen in Erscheinung. Dabei finden sich häufig Systeme zum Monitoring von Patienten im Haushalt und unterwegs, im stationären Bereich oder in Pflegeeinrichtungen sowie ubiquitäre Informationssysteme in medizinischen Einrichtungen und für die Logistik. Diese Systeme können auch der Information und Dokumentation dienen, z. B. für Abrechnungszwecke oder aufgrund gesetzlicher Verpflichtungen (siehe dazu die Einführung in diesen Schwerpunkt sowie PerCoMed Projekt 2007 und Orwat et al. 2008). Konkrete Aussagen über die Kostenstruktur, Wirtschaftlichkeit, Erstattungsfähigkeit oder Finanzierungsmöglichkeiten von Investitionen lassen sich daher nur differenziert nach medizinischen Versorgungssektoren und -formen treffen. Teils sind Pervasive-Computing-Systeme Bestandteil von Diagnose und Therapie oder sie operieren über verschiedene Versorgungssektoren hinweg.
Ferner können sie der Prävention dienen, Bestandteil der Pflege sein oder in das Feld der „Wellness"-Anwendungen fallen. Entsprechend ihrer Zuordnung zu verschiedenen Versorgungssektoren und -formen ergeben sich unterschiedliche Kostenerstattungs- und (Re-) Finanzierungsmöglichkeiten.

Eine weitere Kategorie von Systemen dient der innerbetrieblichen Organisation und dort in erster Linie der Kosteneinsparung oder Einnahmeverbesserung. In diesen Fällen kann der medizinische Leistungserbringer grundsätzlich eine Innovation finanzieren, wenn Kosteneinsparungen und Einnahmeverbesserungen (z. B. durch Fallzahlerhöhungen) dem Leistungserbringer direkt zufallen. Kosteneinsparungen in der innerbetrieblichen Organisation können mit dem Einsatz von Pervasive-Computing-Systemen beispielsweise durch Automatisierung von Prozessen, Verbesserung der Informationsversorgung und der Kommunikation des Personals oder Verbesserung sowie Automatisierung der Dokumentation und der Logistik erreicht werden. Da mit vielen Systemen des Pervasive Computing die Verlagerung der Patientenüberwachung aus dem stationären Sektor in den Heimbereich erreicht werden soll (z. B. mittels Home Monitoring), können Liegezeiten verkürzt werden und u. U. Fallpauschalen besser abgerechnet werden. Wird die Innovation zu Marketingzwecken eingesetzt (etwa mit Forschungsprojekten für den Renommeegewinn oder für die Verbesserung des Images in der Öffentlichkeit), kann sie normalerweise vom medizinischen Leistungserbringer oder durch Sponsoring finanziert werden.

Grundsätzlich könnte die Finanzierung von Pervasive-Computing-Anwendungen über private Zahlungen oder Zuzahlungen erfolgen, woran sich aber Fragen der Versorgungsgerechtigkeit anschließen (Möser et al. 2003, S. 103). Denkbar wären in diesen Fällen Leihmodelle, Leasingmodelle oder der Verkauf von Pervasive-Computing-Systemen an Patienten. Der offensichtliche Nachteil ist aber, dass der Patient, der in der Regel eine Kostenübernahme durch die Krankenkasse erwartet, selber zahlen muss.

Der häufigste Fall wird allerdings sein, dass mit Pervasive-Computing-Innovationen der medizinische Nutzen erhöht werden soll und zusätzliche Kosten anfallen. Dann wird in der Regel die Vergütung durch die Krankenkassen 
Abb. 1: Finanzierungsmöglichkeiten

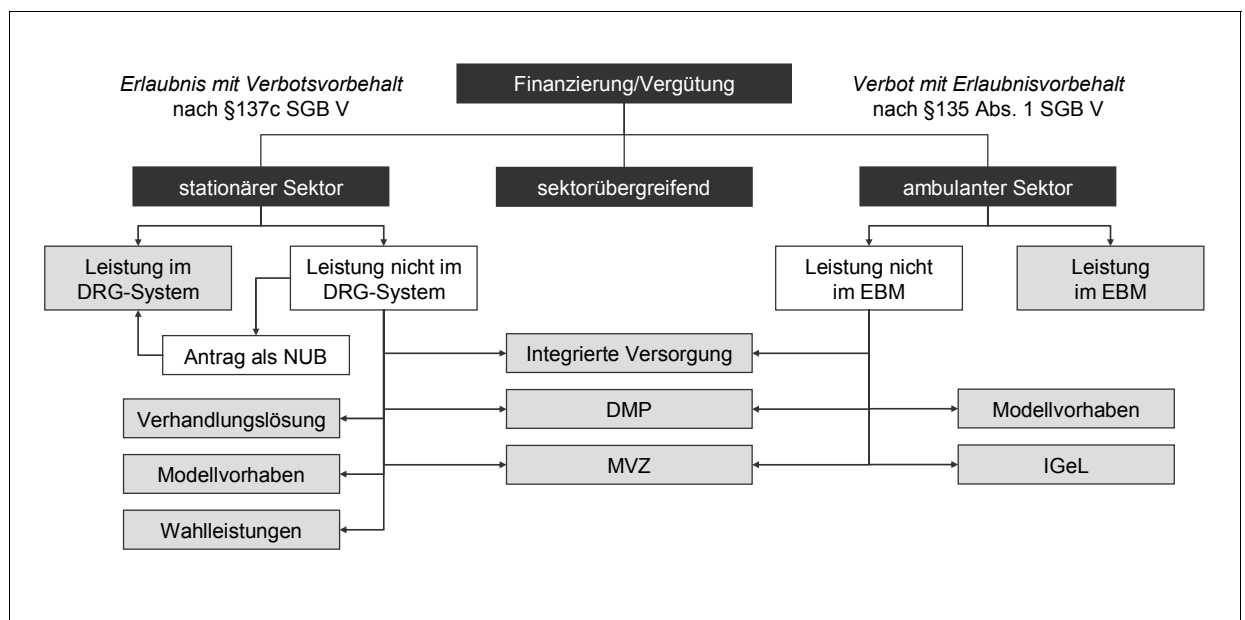

DMP: Disease Management Programme / Strukturiertes Behandlungsprogramm

DRG-System: Diagnosis Related Group System / Fallpauschalensystem

EBM: Einheitlicher Bewertungsmaßstab

IGeL: Individuelle Gesundheitsleistungen

MVZ: Medizinisches Versorgungszentrum

NUB: Neue Untersuchungs- und Behandlungsmethode

SGB: Sozialgesetzbuch

Quelle: Eigene Darstellung

im Rahmen der Gesetzlichen Krankenversicherung (GKV) angestrebt. Dies schließt neue Modelle der medizinischen Versorgung mit ein, wie etwa die integrierte Versorgung, strukturierte Behandlungsprogramme oder medizinische Versorgungszentren. Abbildung 1 liefert eine Übersicht über die im Folgenden näher betrachteten Kostenerstattungsmöglichkeiten. ${ }^{1}$

\section{Möglichkeiten der Kostenerstattung und Finanzierung}

\subsection{Kostenerstattung im stationären Bereich}

In Deutschland gehören zur stationären Versorgung neben den Krankenhäusern auch Vorsorge- und Rehabilitationseinrichtungen. Im Rahmen der GKV sind sie verpflichtet, das fallpauschalierende Entgeltsystem G-DRG (German-Diagnosis Related Group System) anzuwenden, mit dem eine Vergütung der laufenden Kosten auf Basis von diagnosebezogenen Fallpauschalen für bestimmte Fallgruppen (DRGs) erfolgt (siehe u. a. Düllings 2003; Busse, Riesberg 2005). ${ }^{2}$
Bei der Einführung von Innovationen im stationären Bereich gilt grundsätzlich die Erlaubnis mit Verbotsvorbehalt nach $\S 137 \mathrm{c}$ SGB $\mathrm{V}$, wonach alle innovativen Leistungen im Krankenhaus zu Lasten der GKV eingeführt und erbracht werden dürfen - es sei denn, der zuständige Gemeinsame Bundesausschuss (G-BA) hat sie verboten. Werden die Kosten einer Innovation durch die Abrechnungssätze des DRG-Systems abgebildet bzw. abgedeckt, kann die Leistung vollständig darüber abgerechnet werden. Dies kann auch für PervasiveComputing-Systeme gelten, die Bestandteil von anerkannten medizinischen Leistungen sind, und deren Sachkosten dann mit den Abrechnungssätzen gedeckt werden können.

Problematisch wird es, wenn die Kosten von innovativen diagnostischen oder therapeutischen Verfahren nicht durch die DRG-Sätze gedeckt sind, weil entsprechende Ziffern fehlen oder die Abrechnungssätze relativ zu niedrig sind. Dann können entweder Krankenhäuser und Krankenkassen für bestimmte Leistungen zeitlich befristete fall- oder tagesbezogene Entgelte vereinbaren (Verhandlungslösung) oder es können neue Leistungen in Form einer $\mathrm{Neu}$ en Untersuchungs- und Behandlungsmethode $(N U B)$ in das DRG-System mit Hinzufügung 
oder Anpassung der Abrechnungsziffern der Fallpauschalen aufgenommen werden (siehe Abb. 1). Bei Letzteren müssen die Innovationen in wissenschaftlichen Studien überprüft werden, ob sie für eine ausreichende, zweckmäßige und wirtschaftliche Versorgung erforderlich sind, wobei eine Reihe von Nachweisen in Form von Kosten-Nutzen-Analysen notwendig ist. Das Verfahren wird vom G-BA mit Unterstützung durch das Institut für Qualität und Wirtschaftlichkeit im Gesundheitswesen durchgeführt (vgl. z. B. Henke, Reimers 2006; Hornschild et al. 2006, S. 90ff.; BMG 2008). Allerdings wird das Verfahren der Aufnahme von neuen Leistungen als zeit- und kostenaufwendig kritisiert (siehe Abschnitt 3).

\subsection{Wahlleistungen}

Krankenhäuser haben die Möglichkeit, sogenannte medizinische Wahlleistungen anzubieten und diese privat als Selbstzahlerleistung mit dem Patienten abzurechnen (Privatliquidation). Wahlleistungen umfassen in der Regel Leistungen, für die eine medizinische Indikation fehlt (z. B. Schönheitsoperationen), die zur Behandlung der Erkrankung nicht notwendig sind (z. B. homöopathische Begleitbehandlungen) oder die als Alternativleistungen angesehen werden (DKG 2005; Braun et al. 2006, S. 43). Letztere umfassen auch innovative, alternative Untersuchungs- und Behandlungsmethoden, die mehr Kosten verursachen oder nicht sachgerecht als Standardbehandlung abgerechnet werden können. Dies können auch Leistungen sein, in denen Pervasive-Computing-Systeme zur Anwendung kommen. Allerdings ist die Abgrenzung zum Katalog der Regelleistungen in vielen Fällen problematisch. Die Entscheidung ist dann oft einzelfallbezogen, d. h. sie kann dann von Krankenhaus zu Krankenhaus unterschiedlich ausfallen, wodurch eine erhebliche Intransparenz für die Patienten entsteht (DKG 2005).

\subsection{Strukturierte Behandlungsprogramme}

Strukturierte Behandlungsprogramme, die auch als Disease Management Programme (DMPs) bezeichnet werden, wurden mit dem Zweck eingeführt, die Versorgung von Patienten mit chronischen Erkrankungen $\mathrm{zu}$ verbessern unter anderem durch die bessere Zusammenarbeit von Hausarzt, Facharzt, Schwerpunktpraxis, Krankenhaus etc. Sie sind heute allerdings noch auf wenige Krankheiten beschränkt (Diabetes mellitus Typ 1 und 2, koronare Herzkrankheiten, chronisch-obstruktive Lungenerkrankung, Asthma und Brustkrebs). In DMPs können auch Krankenhäuser ambulante Leistungen erbringen (BMG 2008). Im Allgemeinen wird zu den Programmen angemerkt, dass sie zwar die Versorgungsqualität verbessern können, aber auch die Behandlungen durch Richtlinien einschränken könnten. Ebenso können nicht in DMPs eingeschriebene chronisch Kranke benachteiligen werden. Ferner wird der bürokratische Aufwand, insbesondere der erhöhte Dokumentationsaufwand bei Ärzten, kritisiert (Braun et al. 2006, S. 34).

Viele Pervasive-Computing-Systeme dienen dem langfristigen Monitoring von chronischen Erkrankungen. Vor allem die Aufzeichnung von physiologischen Daten über lange Zeiträume wird durch Systeme im Heimbereich und für den mobilen Gebrauch möglich. Damit Pervasive-Computing-Systeme Bestandteil von DMPs werden können, sind allerdings entsprechend aufwendige wissenschaftliche Studien zum Nachweis von Wirksamkeit, Sicherheit und Nutzen erforderlich. Denn auch hier gilt, wie in der ambulanten Versorgung, das ,Verbot mit Erlaubnisvorbehalt" (siehe Näheres im Abschnitt 2.5).

\section{$2.4 \quad$ Integrierte Versorgung}

Die integrierte Versorgung dient insbesondere der Koordination zwischen verschiedenen Leistungserbringern, um eine abgestimmte Versorgung über einzelne Gesundheitssektoren hinweg bieten zu können. $\mathrm{Zu}$ den Vorteilen werden üblicherweise die Vermeidung von Doppeluntersuchungen oder der oft kurzfristige Zugang zu Fachärzten gezählt. Für die Realisierung ist ein Vertrag der integrierten Versorgung nach $\S$ 140 a-e SGB V zwischen den beteiligten Leistungserbringern und den Krankenkassen erforderlich (Henke, Reimers 2006). Mögliche Vertragspartner sind beispielsweise Vertragsärzte, Träger zugelassener Krankenhäuser, stationäre Vorsorge- und Rehabilitationseinrichtungen, 
medizinische Versorgungszentren, stationäre Pflegeeinrichtungen oder Managementgesellschaften. Bei der Vereinbarung von Vergütungsmodalitäten wie Vergütungshöhe, Abrechnungsdetails, Budgetverantwortung, interne Verteilung der finanziellen Mittel etc. gibt es Freiräume, sodass die integrierte Versorgung sich prinzipiell auch für die Einführung von Innovationen durch Kooperationen eignet.

Für Telemedizinanwendungen, zu denen Pervasive-Computing-Anwendungen teilweise zählen, gibt es bereits integrierte Versorgungsverträge. Die Inhalte der Verträge werden allerdings oft nicht transparent gehandhabt, sodass andere Einrichtungen die dabei gewonnenen Erfahrungen nicht nutzen können. Ferner gibt es kaum integrierte Versorgungsverträge mit dem Rettungsdienst, da präklinische Leistungen kaum zu bewerten sind und der Rettungsdienst anderen Finanzierungssystemen unterliegt. $\mathrm{Zu}-$ dem wird gefordert, dass über den gesamten Behandlungsprozess ganzheitliche Fallpauschalen für das gesamte Krankheitsbild notwendig sind, ansonsten werden mit dem bestehenden DRG-System Sektorengrenzen aufgebaut. Dazu wird aber mit Änderungen durch das GKVModernisierungsgesetz gerechnet. ${ }^{4}$

\subsection{Erstattungsmöglichkeiten ambulanter Leistungen}

Für Pervasive-Computing-Anwendungen relevante Erfahrungen aus der Telemedizin zeigen, dass Telemedizin weder als eigenständige medizinische Leistung noch als ein abgrenzbares medizinisches Verfahren anzusehen ist. Telemedizin unterstützt bestehende medizinische Verfahren wie Diagnostik, Befundung oder Konsiliargespräch. Die Abrechnung ist dann nach der Gebührenordnung für Ärzte (GOÄ) oder dem für die Vergütung im Rahmen der GKV maßgeblichen Einheitlichen Bewertungsmaßstab (EBM) möglich. Oft fehlen aber entsprechende Abrechnungsziffern für innovative Leistungen oder die Abrechnungssätze sind so niedrig, dass sie nicht für die Finanzierung von komplexen IT-Lösungen ausreichen (Gründel 2003).

Werden durch Pervasive-ComputingAnwendungen neue diagnostische und therapeutische Verfahren ermöglicht, kann grundsätzlich der EBM angepasst werden. Allerdings gilt bei Innovationen im ambulanten Sektor im Gegensatz zum stationären Sektor das Verbot mit Erlaubnisvorbehalt nach $\S 135$ Abs. 1 SGB V (auch für ambulante Leistungen der Krankenhäuser). Danach dürfen im GKV-System nur dann bestimmte Leistungen zu Lasten der GKV erbracht werden, wenn der G-BA sie ausdrücklich erlaubt hat und in den Leistungskatalog aufgenommen hat. Die Erlaubnis wird bei Nachweis erteilt, dass die Innovation einen medizinischen Nutzen hat, gleichzeitig aber auch notwendig und wirtschaftlich ist. Das Verfahren der Nutzenbewertung wird durch die Verfahrensordnung des G-BA geregelt. Bei positiver Prüfung werden die innovativen medizinischen Leistungen im EBM aufgenommen (z. B. Henke, Reimers 2006, S. 29ff.). Auch für den ambulanten Bereich wird kritisiert, dass dieses Beratungsverfahren zu erheblichen Verzögerungen bei der Einführung von Innovationen führen kann (siehe Abschnitt 3).

\subsection{Individuelle Gesundheitsleistungen}

Niedergelassene Ärzte können neben der Abrechnung von Leistungen der GKV auch Einnahmen aus den sogenannten Individuellen $\mathrm{Ge}$ sundheitsleistungen (IGeL) erzielen. Diese werden als individualvertraglich vereinbarte Gesundheitsleistungen direkt mit dem Patienten nach der GOÄ abgerechnet. Enthalten sind Leistungen, die der G-BA zwar als medizinisch sinnvoll, aber als nicht notwendig klassifiziert hat bzw. deren Qualität und Wirkung nicht oder noch nicht ausreichend gesichert (BMG 2008) und die damit nicht im Leistungskatalog der GKV enthalten sind. Welche Leistungen als IGeL einzustufen sind, wird in mehreren Listen unterschiedlicher Berufsverbände geregelt. Diese Form der Abrechnung ist für PervasiveComputing-Anwendungen u. a. im Fitness- oder Wellnessbereich denkbar, auf die eine Reihe von Anwendungen des Pervasive Computing (z. B. zum langfristigen Monitoring von Vitalparametern) ausgerichtet ist. Ähnlich wie bei den Wahlleistungen im stationären Sektor wird bei den IGeL kritisiert, dass sie zu einer zunehmenden Auslagerungen medizinischer Leistungen in den privat zu zahlenden Bereich führen können (Braun et al. 2006, S. 44). 


\subsection{Modellvorhaben}

Sind medizinische Leistungen nicht in den DRG- oder EBM-Katalogen der Regelleistungen enthalten, können nach $\S 63 \mathrm{ff}$. SGB V stationäre oder ambulante Leistungserbringer $\mathrm{Mo}$ dellvorhaben mit Krankenkassen vereinbaren. Da die Modellvorhaben der Erprobung und Einführung von neuen Behandlungs- und Untersuchungsmethoden, Medizingeräten und auch von Informationssystemen dienen, können sie ein besonderes Leistungsangebot mit PervasiveComputing-Systemen umfassen. In der Regel handelt es sich um Projekte mit einer maximal achtjährigen Laufzeit, die zudem wissenschaftlich von einem unabhängigen Institut begleitet werden müssen. In Modellvorhaben können Leistungen untersucht werden, die der Grundlagenforschung zuzurechnen sind, aber auch die vom G-BA noch nicht zugelassen worden sind oder ausgeschlossen wurden. Üblicherweise sind Modellvorhaben mit einem umfangreichen Verhandlungsprozess zwischen den Krankenkassen und dem Leistungserbringer sowie mit dem Bundesversicherungsamt verbunden (Amelung, Schumacher 2000; Straub 2006).

\subsection{Medizinische Versorgungszentren}

Medizinische Versorgungszentren (MVZ) werden in der Regel für die ambulante und fachübergreifende Zusammenarbeit von Leistungserbringern unterschiedlicher Fachgebiete an bestimmten Orten eingerichtet. Es soll eine gemeinsame Verständigung über Krankheitsverläufe, Therapie- und Behandlungsmethoden erreicht werden. In der Regel stellen sie eine Verhandlungslösung zwischen den medizinischen Leistungserbringern untereinander und mit den Krankenkassen dar (BMG 2008). Da es bereits telemedizinische Versorgungszentren gibt, erscheint diese Versorgungsform auch für Pervasive-Computing-Systeme interessant. Dies gilt insbesondere dann, wenn sie eine Art Telemedizin darstellen wie beispielsweise das „,intelligente“ Telemonitoring mit automatischer Auswertung von physiologischen Parametern und automatischer Alarmierung bei Unregelmäßigkeiten. Allerdings gilt auch für MVZs das „Verbot mit Erlaubnisvorbehalt", wodurch die Bedingungen für Innovationen ähnlich zum ambulanten Bereich ausfallen.

\section{Problemlage}

Der vorangegangene Überblick über Vergütungsformen im deutschen Gesundheitswesen hat gezeigt, dass sich zwar einerseits grundsätzlich Finanzierungsmöglichkeiten der laufenden Kosten für Pervasive-Computing-Systeme finden lassen. Dabei stellte sich im Rahmen von Experteninterviews heraus, dass Branchenangehörige insbesondere die integrierte Versorgung und Modellvorhaben als viel versprechend ansehen (Panova 2008), obwohl beide Formen mit erheblichem Verhandlungs- und Verwaltungsaufwand verbunden sind. Zudem läuft die staatliche Förderung von Vorhaben der integrierten Versorgung Ende 2008 aus. Von den befragten Experten wird zudem eingeschätzt, dass Angebote von Pervasive-Computing-Systemen als Bestandteil von Wahlleistungen oder IGeL auf Ablehnung durch die Patienten stoßen werden. Andererseits wird im Folgenden beschrieben, dass die Implementierung von Pervasive-Computting-Systemen mit einigen Problemen verbunden sein kann, vor allem dann, wenn mit ihnen keine deutliche Kostensenkung verbunden ist. Einerseits kann dies an dem allgemein geltenden Regulierungsrahmen (Abschnitt 3.1) liegen, andererseits an Besonderheiten des Pervasive Computing (Abschnitt 3.2).

\subsection{Der problematische Regulierungsrahmen}

Im Allgemeinen wird kritisiert, dass der Regulierungsrahmen hinsichtlich Vergütungs- bzw. Finanzierungsmöglichkeiten im Gesundheitswesen ein Hemmnis für die Einführung von Innovationen der Medizintechnik darstellt. Dies gilt insbesondere, wenn die Innovation zwar die Qualität, aber auch die Kosten steigert (siehe auch Schmitz-Rode et al. 2005, S. 499ff.; Hornschild et al. 2006, S. 90ff., 116ff.; Straub 2006). Der komplexe, sich ständig wandelnde Regulierungsrahmen (Medizinproduktrecht ${ }^{3}$, Leistungskataloge, etc.) kann zu Belastungen für medizinische Leistungserbringer führen, wenn Finanzierungsmöglichkeiten gesucht, ausgehandelt 
und realisiert werden sollen. Insbesondere die Verfahren der Aufnahme in die Vergütungskataloge DRG und EBM stellen aus Sicht der Technologieanbieter einen zeit- und kostenaufwendigen „trial-and-error“-Prozess mit hohen Unsicherheiten dar und können insbesondere für kleine und mittelständische Technologieanbieter oder junge Unternehmen effektive Marktzutrittsbarrieren darstellen. ${ }^{5}$ Oft wird der lange Weg von der Produktidee bis zum Rückfluss des eingesetzten Kapitals kritisiert, der sich über Prototyp, Kleinserie, vorklinische Erprobung, klinischer Studie, gesundheitsökonomische Evaluierung durch Einrichtungen der Health Technology Assessment, Zulassung und Erlangung der Erstattungsfähigkeit in der GKV ziehen kann. Die Laufzeit allein der klinischen Studien wird mit sechs Monaten bis zwei Jahren angegeben (z. B. Schmitz-Rode et al. 2005, S. 521). Sie verursachen zudem hohe Kosten. Für die formelle Aufnahme einer Innovation in das DRG-System wird von manchen Autoren ein Zeitraum von mindestens zwei Jahren genannt, der aber auch länger ausfallen kann; zudem muss eine relativ große Zahl von Krankenhäuser beteiligt sein (Schlottmann 2002; Hornschild et al. 2006, S. 101; Neubauer, Ujlaky 2006).

Ferner bedeuten neue Formen der Leistungserbringung und -vergütung (wie z. B. die integrierte Versorgung, Verhandlungslösungen mit Krankenkassen, die Errichtung Medizinischer Versorgungszentren) für die medizinischen Leistungserbringer einen gesteigerten Verwaltungsaufwand sowie einen erheblichen Verhandlungsaufwand zwischen den verschiedenen beteiligten Akteuren.

Allerdings sind diese Kritikpunkte vor dem Hintergrund zu sehen, dass in der medizinischen Versorgung im Allgemeinen der nachgewiesene medizinische Nutzen gegenüber dem ökonomischen Nutzen höher eingestuft wird. Unter Umständen kann sich der lange Prozess auch insofern positiv auswirken, dass in der Zwischenzeit neue medizinische Erkenntnisse gefunden werden, die etwa die Schädlichkeit der Innovation nachweisen (Stuppardt 2007) oder dass andere Technologien sich als besser oder als de facto Standard herausstellen (Christensen, Remler 2007).

\subsection{Die besonderen Eigenschaften des Pervasive Computing}

Eine Reihe von besonderen Charakteristika des Pervasive Computing legt die Annahme nahe, dass die Einführung und Finanzierung von Systemen des Pervasive Computing problematischer sein kann, als die Einführung anderer Medizintechnik. Auch international wird berichtet, dass bei der Einführung von Pervasive Computing Finanzierungshürden aufgrund problematischer Kostenerstattung auftreten (Fisher 2004; Wan, Taveras 2007, S. 295). Als bestimmend sind folgende spezifischen Eigenschaften anzuführen:

1. Bei vielen Systemen des Pervasive Computing handelt es sich nicht um medizintechnische Großgeräte einzelner Hersteller. Pervasive-Computing-Systeme sind in vielen Fällen aus konventionellen Geräten und Netzwerken unterschiedlichster Hersteller zusammengesetzt (wie z. B. Displays, PDAs, Mobiltelefonen und WLANs). Zudem sind einige Technologieanbieter nicht auf Medizintechnik spezialisiert. Dadurch, dass ein großer Investor fehlen kann, ist es u. U. fraglich, ob klinische Studien finanzierbar sind. Kann für die klinischen Studien eine Verbundlösung gefunden werden, stellt sich häufig die Frage, wer für die Durchführung und Finanzierung der Studien und letztendlich für die Systemeinführung die Verantwortung übernimmt.

2. Bei einer Reihe von Pervasive-ComputingSystemen ist vorgesehen, dass sie mobil beim Patienten getragen werden, d. h. es handelt sich oft nicht um einzelne medizintechnische Geräte, die bei bestimmten Leistungserbringern lokalisiert sind, die dann meist auch die Investoren sind. Stattdessen erbringen die mobilen Pervasive-Computing-Anwendungen oft gleichzeitig Nutzen bei mehreren Akteuren aus verschiedenen Einrichtungen oder Versorgungssektoren (z. B. bei Patienten, Ärzten, Krankenkassen, Krankenhäusern), sodass Kooperationslösungen mit einem hohen Verhandlungsaufwand verbunden sind. Besonders problematisch wird es, wenn die Akteure aus unterschiedlichen Regionen mit voneinander abweichenden regulativen Rahmenbedingungen stammen. 
3. In vielen Fällen wird der ökonomische Nutzen der Pervasive-Computing-Systeme erst bei Erreichen von Netzwerkeffekten deutlich und quantifizierbar. ${ }^{6}$ Dies bedeutet, dass er erst bei der vernetzten Anwendung in großer Zahl deutlich wird, allerdings kaum bereits bei Prototypen oder in Kleinserientests. Zudem ergeben sich viele Vorteile erst bei der Anbindung an bestehende Krankenhausinformationssysteme (z. B. durch Automatisierung der Dokumentation). Die Anbindung ist mit zusätzlichen Kosten verbunden, die selten bereits bei den Prototyp-Tests deutlich genug abgeschätzt werden können.

4. Da Pervasive-Computing-Systeme in vielen Fällen auf konventionellen, bereits eingeführten Komponenten beruhen, können zwar einerseits oft niedrige Stückkosten für einzelne Systemkomponenten vorliegen (z. B. für einzelne RFID-Tags). Andererseits ist aber u. U. mit hohen Kosten für die gesamte Infrastruktur zu rechnen (wie z. B. bei der Anschaffung der erforderlichen Lesegeräte). Zudem können sich die geringen Stückkosten bei der Einführung für ganze Patientengruppen oder durchgehend in der ganzen Organisation stark summieren.

\section{Handlungsempfehlungen}

Aus den obigen Ausführungen lässt sich eine Reihe von Handlungsempfehlungen ableiten. Entwickler, Hersteller und Technologieanbieter könnten sich stärker auf Systeme fokussieren, die keine oder kaum zusätzliche Kosten gegenüber bestehenden Verfahren verursachen und die in erster Linie auf Kostenreduzierung und Verbesserung der organisationsinternen Prozesse oder der Dokumentation abzielen. Dadurch könnten die üblichen aufwendigen $\mathrm{Zu}$ lassungs- und Aufnahmeverfahren umgangen werden, die bei den auf Diagnose und Therapie zielenden Systemen anfallen. Sollen PervasiveComputing-Systeme dennoch Bestandteil der Diagnose und Therapie sein, scheinen Systeme vorteilhaft zu sein, die in medizinischen Regelleistungen existierende Technologien mit deutlichen Kostensenkungen bei (mindestens) gleichem medizinischem Nutzen ersetzen. Allerdings ist insbesondere für das medizinische Personal der Nachweis einer Steigerung des medizinischen Nutzens unumgänglich für die Akzeptanz der Systeme, so ein zentrales Ergebnis der PerCoMed-Akzeptanzanalyse (siehe Wölk et al. in diesem Heft).

Eine Fokussierung auf Systeme, die Bestandteil von medizinischen Leistungen sind, und die entweder als Wahlleistung im stationären Bereich oder als Individuelle Gesundheitsleistung im ambulanten Bereich über die Privatliquidation an Patienten veräußert werden, kann zwar auch als mögliche Finanzierungsstrategie gesehen werden. Allerdings stößt diese Form an die ethischen Grenzen des Erhalts der Versorgungsgerechtigkeit, wenn sie einen Trend unterstützen würde, medizinische Leistungen aus den Katalogen der Regelleistungen herauszunehmen oder gar nicht erst aufzunehmen (z. B. Braun et al. 2006).

Die Probleme der aufwendigen klinischen Studien, aber auch des problematischen Nachweises des wirtschaftlichen Nutzens kann mit der öffentlichen Finanzierung von Studien, Forschungs- und Pilotprojekten und der frühen Implementierungsschritte gemindert werden. Der zunehmende Verhandlungsaufwand, den medizinische Leistungserbringer $\mathrm{zu}$ tragen haben, kann durch vermehrte Transparenz hinsichtlich der erzielten Verhandlungslösungen reduziert werden. Insbesondere sollen Lerneffekte bei anderen Akteuren erreicht werden. Beispielsweise können Informationen, Modellverträge oder Standards öffentlich bereitgestellt werden; sie könnten zeigen, wie Vorhaben zur integrierten Versorgung, Modellvorhaben oder Verhandlungslösungen realisiert wurden und welche Erfahrungen dabei anfielen.

Ebenso sollte die Weiterentwicklung der Vergütungssysteme zur besseren Berücksichtigung von Innovationen in den Katalogen der Regelleistungen voranschreiten. In den letzten Jahren ist ansatzweise auf die Kritik, dass Innovationshemmnisse bestehen, reagiert worden und es wurde beispielsweise die systematische Evaluierung in Form der Health Technology Assessment auch in Deutschland institutionalisiert (Straub 2006) oder eine Innovationsklausel in das DRG-System eingebaut (BVMed 2006). Danach können Krankenhäuser und Krankenkassen bereits dann eine Verhandlungslösung über eine Neue Untersuchungs- und Behandlungsmethode (NUB) erzielen, wenn die reguläre Aufnahme in das DRG-System noch läuft. 
Die NUB kann dann später über das DRGSystem verrechnet werden (siehe Abbildung 1). Auch die Kassenärztliche Bundesvereinigung hat eine Initiative zur rascheren Einführung patientenrelevanter Innovationen in die vertragsärztliche Versorgung gestartet (KBV 2005). Eine Beurteilung derartiger Maßnahmen steht allerdings bisher noch aus.

\section{Anmerkungen}

1) Es werden vor allem die Kostenerstattungsmöglichkeiten im Rahmen der GKV berücksichtigt, ferner die zwei Modelle Wahlleistungen und Individuelle Gesundheitsleistungen. Beispielsweise bleiben im Folgenden die Investitionen für Gebäude und Ähnlichem im Rahmen der dualen Finanzierung im stationären Bereich unberücksichtigt.

2) Nach einer Übergangsfrist soll ab 2009 das DRG-System das einzige Preissystem für laufende Ausgaben sein und damit das bisherige Mischvergütungssystem aus Pflegesätzen, Fallpauschalen und Sonderentgelten ersetzen.

3) Ein Großteil der Pervasive-Computing-Systeme fällt in diverse Kategorien des Medizinproduktegesetz, das das Inverkehrbringen von Medizinprodukten im Europäischen Wirtschaftsraum mit Vergabe des CE-Kennzeichens regelt (wie z. B. bei medizinischer Elektronik und Elektromedizin, medizinischer Datenverarbeitung oder allgemeinen medizinischen Behandlungseinrichtungen, Geräten und Hilfsmitteln. Die Anforderungen an die erforderlichen klinischen Studien werden im Vergleich zur Aufnahme in die Leistungskataloge als geringer eingeschätzt (Stuppardt 2007, S. 847f.).

4) Vgl. dazu die Stellungnahmen von Teilnehmern des PerCoMed-Expertenworkshops (PerCoMed Projekt 2007, S. 11f.).

5) Vgl. zu diesen Zutrittsbarrieren die Angaben bei Hornschild et al. 2006, S. 96, 124

6) Vgl. auch für Informations- und Kommunikationstechnologien im Allgemeinen Hornschild et al. 2006; Christensen, Remler 2007.

\section{Literatur}

Amelung, V.E.; Schumacher, H., 2000: Managed Care - Neue Wege im Gesundheitsmanagement. Wiesbaden

BMG - Bundesministerium für Gesundheit, 2008: Die Gesundheitsreform - Webangebot des Bundesministeriums für Gesundheit; http://www.diegesundheitsreform.de (download April 2008)
Braun, B.; Reiners, H.; Rosenwirth, M. et al., 2006: Anreize zur Verhaltenssteuerung im Gesundheitswesen. Effekte bei Versicherten und Leistungsanbietern. Chartbook. Gütersloh: Bertelsmann Stiftung, Themenfeld Gesundheit; Universität Bremen, Zentrum für Sozialpolitik; http://www.bertelsmannstiftung.de/bst/de/media/Chartbook_Anreize.pdf (download 7.5.08)

Busse, R.; Riesberg, A., 2005: Gesundheitssysteme im Wandel - Deutschland. Kopenhagen: WHO, Regionalbüro für Europa im Auftrag des Europäischen Observatoriums für Gesundheitssysteme und Gesundheitspolitik; http://www.euro.who.int/docume nt/e85472g.pdf (download 7.5.08)

BVMed - Bundesverband Medizintechnologie e.V., 2006: Einführung innovativer und neuer Medizinprodukte im G-DRG-System. Berlin

Christensen, M.C.; Remler, D.K., 2007: Information and communications technology in chronic disease care: Why is adoption so slow and is slower better? NBER Working Paper Series, No. 13078, Cambridge, MA: National Bureau of Economic Research

DKG - Deutsche Krankenhausgesellschaft, 2005: Informationspapier der Deutschen Krankenhausgesellschaft zum Angebot medizinischer Wahlleistungen durch Krankenhäuser. Berlin; http://www.dkgev. de/pdf/657.pdf (download 7.5.08)

Düllings, J., 2003: Einführung des DRG-Systems und seine Auswirkungen auf die Krankenhausplanung. In: Thiele, G. (Hg.): Einführung der DRGs in Deutschland, Heidelberg, S. 1-20

Fisher, R., 2004: New age technology [telecare monitors for aged people]. In: The Engineer 293/7655 (2004), S. 22-26

Gründel, M., 2003: Telemedizin als vergütungsrechtliches Niemandsland? In: Niederlag, W.; Burchert, H.; Lemke, H.U. (Hg.): Telemedizin und Ökonomie. Ökonomische Effekte, Abrechnungsmodalitäten und Geschäftsmodelle. Health Academy 01/2003, Dresden: Dresden-Friedrichstadt General Hospital, S. 108-112

Henke, K.D.; Reimers, L., 2005: Finanzierung, Vergütung und Integrierte Versorgung im medizinischtechnischen Leistungsgeschehen. Berlin; http://www.spectaris.de/downloads/presse/zukunftsfo rum_05/ZF05_Studie_191005.pdf(download 7.5.08)

Hornschild, K.; Raab, S.; Weiss, J.-P., 2006: Die Medizintechnik am Standort Deutschland - Chancen und Risiken durch technologische Innovationen, Auswirkungen auf und durch das nationale Gesundheitssystem sowie potenzielle Wachstumsmärkte im Ausland. Forschungsprojekt im Auftrag des Bundesministeriums für Wirtschaft und Arbeit, 2., neubearbeitete und erweiterte Auflage. Berlin: DIW in 
Zusammenarbeit mit VDI Technologiezentrum; http://www.diw.de/deutsch/produkte/publikationen/ diwkompakt/docs/diwkompakt_2005-010.pdf (download 7.5.08)

KBV - Kassenärztliche Bundesvereinigung, 2005: Eine Initiative zur rascheren Einführung patientenrelevanter Innovationen in die vertragsärztliche Versorgung. Manual. Berlin: Innovationsservice der KBV

Möser, K.; Milde, S.; Haroske, G. et al., 2003: Abrechnungsmodalitäten telemedizinischer Leistungen. In: Niederlag, W.; Burchert, H.; Lemke, H.U. (Hg.): Telemedizin und Ökonomie. Ökonomische Effekte, Abrechnungsmodalitäten und Geschäftsmodelle. Health Academy 01/2003, Dresden: Dresden-Friedrichstadt General Hospital, S. 99-107

Neubauer, G.; Ujlaky, R., 2006: Finanzmanagement in Krankenhäusern. In: Busse, R.; Schreyögg, J.; Gericke, C. (Hg.): Management im Gesundheitswesen. Heidelberg, S. 232-242

Orwat, C.; Graefe, A.; Faulwasser, T., 2008: Towards Pervasive Computing in Health Care - A Literature Analysis. In: BMC Medical Informatics and Decision Making (eingereicht)

Panova, V., 2008: Refinanzierungsmodelle von Pervasive Computing Systemen im deutschen Gesundheitswesen, am Beispiel der Fallstudie „MS Nurses“" (Unveröffentlichte Diplomarbeit). Karlsruhe: Forschungszentrum Informatik, ITAS im Forschungszentrum Karlsruhe

PerCoMed Projekt, 2007: Bericht zum PerCoMedExpertenworkshop. PerCoMed Report (Projekt PerCoMed - Pervasive Computing in der medizinischen Versorgung), Karlsruhe: ITAS im Forschungszentrum Karlsruhe; http://www.percomed.de/images/stori es/expertenworkshop/percomed_workshopbericht_fi nal.pdf (download 7.5.08)

Schlottmann, N., 2002: Die Anpassung der ARDRGs und ihre Grenzen. In: Das Krankenhaus 94/1 (2002), S. 26-33; http://www.dkgev.de/pdf/295.pdf (download 7.5.08)

Schmitz-Rode, T.; Floren, M.G.; Baumann, M., 2005: Schlüsseltechnologien und deren Potentiale für die Medizintechnik - Implementierungsfaktoren und Substitutionspotentiale von Innovationen. In: Farkas, R.; Becks, T. (Hg.): Studie zur Situation der Medizintechnik in Deutschland im internationalen Vergleich. Aachen: Aachener Kompetenzzentrum Medizintechnik et al., S. 494-534; http://akm-aachen.de/fileadmin /user_upload/MTStudie.pdf (download 7.5.08)

Straub, C., 2006: Der Weg von Innovationen in die Praxis: Das Stent-Projekt der Techniker Krankenkasse. In: Häussler, B.; Paquet, R.; Klusen, N. (Hg.): Jahrbuch der medizinischen Innovationen.
Band 2: Gesundheit im Jahr der Innovation. Stuttgart, S. 51-57

Stuppardt, R., 2007: Innovationen und ihre Perspektiven im Spannungsfeld zwischen Finanzierung und Versorgungsfortschritt im Gesundheitswesen. In: Ulrich, V.; Ried, W. (Hg.): Effizienz, Qualität und Nachhaltigkeit im Gesundheitswesen. Theorie und Politik öffentlichen Handelns, insbesondere der Krankenversicherung. Baden-Baden, S. 839-854

Wan, D.; Taveras, L.E., 2007: The business of pervasive healthcare. In: Bardram, J.E.; Mihailidis, A.; Wan, D. (Hg.): Pervasive Computing in Healthcare, Boca Raton, FL, S. 275-298

\section{Kontakt}

Dr. Carsten Orwat

Veneta Panova

Forschungszentrum Karlsruhe in der HelmholtzGemeinschaft Institut für Technikfolgenabschätzung und Systemanalyse (ITAS) Postfach 36 40, 76021 Karlsruhe Tel.: +49 (0) 7247 / 82 - 6116 E-Mail: orwat@itas.fzk.de 